\title{
Unveiling the Diverse Spectrum of Fungal Rhinosinusitis
}

\author{
Seok Hyun Cho (D) \\ Department of Otorhinolaryngology-Head and Neck Surgery, Hanyang University College of Medicine, Seoul, Korea
}

Fungi are a ubiquitous and a well-known biological factor contributing to chronic rhinosinusitis with or without nasal polyps. Depending on the level of host immunity, fungal colonization may result in indolent fungus balls or invasive fungal rhinosinusitis [1]. Furthermore, if an individual is allergic to specific fungi, allergic fungal rhinosinusitis can occur, accompanied by severe polyposis and thick eosinophilic mucin. The two noninvasive types of fungal colonization (fungus balls and allergic fungal rhinosinusitis) show racial differences in prevalence, but the reason for this is not yet known [2]. In healthy individuals, the fungus lives in mutualistic symbiosis in the airway and constantly stimulates the mucosal epithelium in specific patterns. However, fungi can cause a variety of diseases and become harmful or hostile parasites in pathological conditions.

The microbial environment in the paranasal sinuses is a bioecosystem consisting of diverse components, namely the bacteriome, fungiome, and virome [3]. Many factors-such as viral infections, antibiotic use, and host stress - can lead to dysbiosis, in which the internal microbial equilibrium (communication) is broken down and the outgrowth of some pathogens occurs. Although the underlying causes remain unknown, fungal dysbiosis and altered host-environmental interactions can cause fungal diseases.

Fungus balls are the most common type of noninvasive fungal rhinosinusitis in Asian populations [4]. Aspergillus species cannot invade the sinus mucosa when the host immunity is competent. Fungus balls are usually unilateral and located in the maxillary sinus. If intralesional hyperdensity (calcification) is observed on computed tomography, we can suspect the presence of fungus balls [5]. In addition, older age and specific lesion morphology (fuzzy or full) increase the diagnostic accuracy. Perhaps the most interesting question regarding fungus balls is how fungi grow in the maxillary sinus, avoiding host immunity.

Contrary to our expectations, fungi exhibit very complex behaviors [6]. Recently, Kim et al. [7] reported that fungus balls are a subtype of bioballs, which consist of four phenotypes (fungus ball, bacterial ball, mixed ball, and double ball) [7]. These types appear similar; therefore, it is not possible to discriminate them during endoscopic sinus surgery. If a Gram stain shows bacterial colonies in the core of bioballs, they can be classified as bacterial balls or mixed balls. Moreover, immunocompromised patients are prone to mixed balls, which are associated with decreased epithelial barrier function. Therefore, further research into this topic is necessary, and we should observe fungal behavior more closely.

\section{CONFLICT OF INTEREST}

No potential conflict of interest relevant to this article was reported.

\section{ORCID}

Seok Hyun Cho https://orcid.org/0000-0001-8218-5894

\section{REFERENCES}

1. Montone KT. Pathology of fungal rhinosinusitis: a review. Head Neck Pathol. 2016 Mar;10(1):40-6.

2. Montone KT, Livolsi VA, Feldman MD, Palmer J, Chiu AG, Lanza DC, et al. Fungal rhinosinusitis: a retrospective microbiologic and pathologic review of 400 patients at a single university medical center. Int J Otolaryngol. 2012;2012:684835.

3. Huffnagle GB, Noverr MC. The emerging world of the fungal microbiome. Trends Microbiol. 2013 Jul;21(7):334-41.

4. Das A, Bal A, Chakrabarti A, Panda N, Joshi K. Spectrum of fungal rhinosinusitis; histopathologist's perspective. Histopathology. 2009 Jun;54(7):854-9.

5. Cha H, Song Y, Bae YJ,WonTB, Kim JW, Cho SW, et al. Clinical char-

Copyright (C) 2020 by Korean Society of Otorhinolaryngology-Head and Neck Surgery.

This is an open-access article distributed under the terms of the Creative Commons Attribution Non-Commercial License (https://creativecommons.org/licenses/by-nc/4.0)

which permits unrestricted non-commercial use, distribution, and reproduction in any medium, provided the original work is properly cited. 
acteristics other than intralesional hyperdensity may increase the preoperative diagnostic accuracy of maxillary sinus fungal ball. Clin Exp Otorhinolaryngol. 2020 May;13(2):157-63.

6. Kim DK, Wi YC, Shin SJ, Jang YI, Kim KR, Cho SH. Bacterial ball as an unusual finding in patients with chronic rhinosinusitis. Clin Exp Otorhinolaryngol. 2018 Mar;11(1):40-5.

7. Kim DK, Wi YC, Shin SJ, Kim KR, Kim DW, Cho SH. Diverse phenotypes and endotypes of fungus balls caused by mixed bacterial colonization in chronic rhinosinusitis. Int Forum Allergy Rhinol. 2019 Nov;9(11):1360-6.

Received December 11, 2019 Accepted January 2, 2020 